\title{
Volatile Compounds from Must and Wines from Five White Grape Varieties
}

\author{
Amélie Slegers ${ }^{1,2}$, Paul Angers ${ }^{2}$ and Karine Pedneault ${ }^{1-3^{*}}$ \\ ${ }^{1}$ Centre de Développement Bioalimentaire du Québec, La Pocatière, QC, GOR 1ZO, Canada \\ ${ }^{2}$ Department of Food Science, Université Laval, Québec, QC, G1V 0A6, Canada \\ ${ }^{3}$ Department of Science, Université Sainte-Anne, Church Point, NS, BOW 1MO, Canada
}

\section{Correspondence to:}

Karine Pedneault

Department of Science, Université Sainte-Anne Church Point, NS, B0W 1M0, Canada

Tel: +1902-769-2114 ext. 7268

E-mail: karine.pedneault@usainteanne.ca

Received: November 30, 2016

Accepted: January 16, 2017

Published: January 18, 2017

Citation: Slegers A, Angers P, Pedneault K. 2017. Volatile Compounds from Must and Wines from Five White Grape Varieties. $J$ Food Chem Nanotechnol 3(1): 8-18.

Copyright: () 2017 Slegers et al. This is an Open Access article distributed under the terms of the Creative Commons Attribution 4.0 International License (CC-BY) (http://creativecommons. org/licenses/by/4.0/) which permits commercial use, including reproduction, adaptation, and distribution of the article provided the original author and source are credited.

Published by United Scientific Group

\section{Abstract}

Viticulture and wine production in challenging environments such as cold and humid areas has substantially expanded in recent years, mostly due to the development of grapevine varieties that show high resistance to fungal diseases and cold temperatures. These interspecific hybrid Vitis (IHV) varieties result from multiple crosses between Vitis vinifera varieties and North American native species such as $V$. riparia and $V$. labrusca. Limited scientific information is available on the aroma and winemaking of IHV, and about the relationships between grape and wine composition in regard to compounds of enological interest. In this work, the profile of volatile compounds of grapes and wines from five white IHV varieties largely grown in cold-climate viticulture (Frontenac blanc, Frontenac gris, Seyval, St. Pepin and Vidal) was determined by GC-MS-SPME. Compound classes detected in juice and wine included fatty acid degradation products that represented the largest part of volatile compounds, fatty acid ethyl esters, terpenes, $\mathrm{C}_{13}$-norisoprenoids, and volatile phenols and other phenyl derivatives. The flavor profile of Vidal showed the largest diversity of compounds in both juice and wine, and the overall highest amount of volatile compounds of all varieties. Principal component analysis showed relationships between the presence of certain $\mathrm{C}_{6}$ compounds, terpene and volatile phenols in musts and in the finished wines.

\section{Keywords}

Vitis, GC-MS-SPME, Volatile compounds, Northern viticulture, Cold tolerance, Interspecific hybrid, Quebec, Canada, Seyval, St. Pepin, Vidal, Frontenac gris, Frontenac blanc

\section{Introduction}

Predictions are that the global wine market will have reached a value of more than $\$ 304$ billion in 2016 [1]. The economic contribution of the wine industry is significant in several sectors such as agriculture, bio-food processing, trade, manufacturing and tourism, making it a powerful tool for regional development. Wine production, which was previously restricted to areas showing suitable environmental conditions for Vitis vinifera varieties such as Pinot noir and Riesling, has become a universal economic activity. Indeed, quality wines are now produced in many areas showing challenging growing conditions such as Eastern Canada, Northern Europe, and Northern Asia [2]. Wine production in challenging cold and humid climate conditions experienced a major boom in recent years with the arrival on the market of interspecific hybrid Vitis (IHV) varieties [3]. These varieties are obtained from interspecific crosses between American native Vitis species (Vitis riparia, V. labrusca, V. rupestris, $V$. lincecumii) and European $V$. vinifera 
varieties. IHV varieties are productive, early-maturing, well adapted to cold temperature, and highly resistant to fungal diseases, making them suitable for cultivation in environments that are unsuited for the implementation of traditional $V$. vinifera varieties [4]. The province of Quebec is the third largest wine region in Canada, and has over 800 hectares devoted to grape production [5]. The Quebec wine industry is nearly $100 \%$ based on IHV varieties, and local growers grow about 50 different IHV varieties. White wines account for $52 \%$ of wine produced in Quebec from more than 20 different white grape varieties, but the main ones are the American varieties Frontenac blanc, Frontenac gris, and St. Pepin, and the French-American varieties Seyval blanc and Vidal.

In recent years, the Quebec wine industry has developed substantially and the quality of local wines became increasingly higher. However, despite these improvements, the lack of detailed knowledge about the oenological characteristics of IHV varieties continues to hinder the development of the industry. Indeed, unlike the traditional $V$. vinifera varieties, whose chemical composition has been widely studied [6-8], knowledge about the chemical composition and oenological potential of IHV varieties remains very limited. In addition, unique genetics confer biochemical characteristics to these varieties that largely differ from those of traditional European varieties, which highly complicates the adaptation of traditional wine production methods [2].

A limited number of studies have focused on the volatile compounds from grapes and wines of IHV varieties. Vidal, the main variety used for ice-wine production in Canada, is one of the few IHV varieties that have been studied [9]. Analysis of Vidal wine aroma by gas chromatography coupled to olfactometry (GC-O) and mass spectrometry (MS) showed that ethyl hexanoate, ethyl octanoate, cis-rose oxide, linalool, geraniol, $\beta$-damascenone and 2 -phenylethanol have a significant olfactory impact on wine aroma $[9,10]$. In a comparative study, Chisholm et al. [11] found that wines from the white IHV varieties Cayuga, Vidal and Seyval blanc had higher concentrations of undesirable volatile aroma compounds than the $V$. vinifera variety Riesling. On the other hand, studies conducted on the IHV variety Traminette showed that the concentrations of monoterpenes in berries was twice higher than in Riesling and Gewürztraminer berries grown under similar environmental conditions [12].

Data on white IHV varieties grown in northern conditions are scarce. In Quebec, a few studies focused on the impact of different agricultural practices on parameters such as cold tolerance [13], disease susceptibility [14], maturity and fruit quality [15], but most studies focused on agronomic aspects rather than wine production. Yet knowledge of the volatile compounds of Vitis varieties is crucial for optimizing the quality of wines. In this context, the aim of the present study was to determine the profiles of volatile compounds in berries and wines from five white IHV varieties (Frontenac blanc, Frontenac gris, Seyval blanc, St. Pepin and Vidal) grown in Quebec, using solid phase microextraction (SPME) coupled with GC-MS, and to establish correlations between the volatile compounds of berry juice and wine.

\section{Materials and Methods}

\section{Samples}

Samples (four to five biological replicates per grape variety) of the white IHV varieties Frontenac blanc and Frontenac gris [colorless mutants from red Frontenac (Vitis riparia $\mathrm{x}$ Landot 4511)], Seyval blanc (Seibel 5656 x Seibel 4986), St. Pepin [(Minnesota 78 x Seibel 1000) x Seyval blanc)], and Vidal (St. Emilion x Seibel 4986) were harvested at commercial maturity, in vineyards located in the Montérégie (45 $7^{\circ} \mathrm{N}$, $72^{\circ} 48^{\prime} \mathrm{W}$ ) and Estrie ( $45^{\circ} 26^{\prime} \mathrm{N}, 72^{\circ} 53^{\prime} \mathrm{W}$ ) areas (Quebec, Canada), during the 2012 season. Samples (50 kg) were preserved at $4{ }^{\circ} \mathrm{C}$ during transportation from the vineyard to the experimental winery.

\section{Reagents and standards}

Absolute ethanol was purchased from Commercial Alcohols (Brampton, ON), and L-tartaric acid and sodium chloride $(\mathrm{NaCl})$ from Fisher Scientific (Fair Lawn, NJ). Deuterated standards ( $\mathrm{d}_{8}$-ethyl acetate, ethyl 4,4,4- $\mathrm{d}_{3}$ butanoate, $\mathrm{d}_{5}-2,3,4,5,6$-benzyl alcohol, 2-phenyl- $\mathrm{d}_{5}$-ethanol, $\mathrm{d}_{11}$-hexanoic acid, $\mathrm{d}_{15}$-ethyl octanoate and $\mathrm{d}_{13}$-hexanol) were purchased from $\mathrm{C} / \mathrm{D} / \mathrm{N}$ Isotopes Inc. (Pointe-Claire, QC). $\beta$-myrcene was purchased from MP Biomedicals (Santa Ana, CA). Ethyl hexanoate and ethyl propanoate were purchased from $\mathrm{Nu}$-Chek-Prep (Elysian, MN). Ethyl vanillate and nonanal were purchased from Alfa Aesar (Heysam, England). Other reagents and standards were purchased from SigmaAldrich (St. Louis, MO) [3].

\section{Technological parameters of musts}

For each sample, 200 berries were randomly picked from 15 different randomly selected clusters. Grape juice was manually extracted by pressing berries in food grade polyethylene bags. Total soluble solids (TSS, ${ }^{\circ}$ Brix), $\mathrm{pH}$ and titratable acidity (TA; g tartaric acid eq./L) were measured on fresh must according to Amerine and Ough [16]. Yeast assimilable nitrogen (YAN) concentration was determined by measuring ammoniacal nitrogen $\left(\mathrm{NH}_{3}\right.$ and $\left.\mathrm{NH}_{4}^{+}\right)$and primary amino nitrogen, as described by Slegers and coworkers [3].

\section{Winemaking}

Samples were stemmed, pressed, treated with potassium metabisulphite $\left(\mathrm{SO}_{2} ; 30 \mathrm{mg} / \mathrm{L}\right)$, and placed at $10{ }^{\circ} \mathrm{C}$ for 8 to 12 hours to allow the sedimentation of the solid residue. Must was then racked, tempered at $18{ }^{\circ} \mathrm{C}$ and inoculated with Saccharomyces cerevisiae var. bayanus Lalvin QA23 $(250 \mathrm{mg} / \mathrm{L}$; Lallemand, Montreal, QC). Fermentations were carried out at $18{ }^{\circ} \mathrm{C}$, with daily monitoring of must density and temperature, until dryness (specific gravity of $0.998 \mathrm{~kg} / \mathrm{L}$ ). Wines were racked, treated with $50 \mathrm{mg} / \mathrm{L} \mathrm{SO}_{2}$, stored at $10{ }^{\circ} \mathrm{C}$ in stainless steel kegs and aged for 5 months. After aging, wines were sulfited, filtered ( $45 \mu \mathrm{m}$ filters, Buon Vino Super Jet Wine, Cambridge, ON), bottled, and kept in the dark at $10{ }^{\circ} \mathrm{C}$ until analyzes that were performed a few days later. Each grape sample (four to five biological replicates per variety) was individually processed and fermented. 


\section{Basic wine composition}

$\mathrm{TA}, \mathrm{pH}$, and ethanol levels were measured according to Amerine and Ough [16]. Volatile acidity (VA) and glycerol concentrations were determined by UV-visible spectroscopy, using commercial test kits, as described by Slegers and coworkers [3].

\section{Analysis of volatile compounds in juice and wine}

Juice and wine volatile compounds were extracted by headspace solid-phase microextraction (SPME) and analyzed by gas chromatography coupled to a time-of-flight mass spectrometer (GC-TOF-MS), as detailed by Slegers et al. [3]. Juice samples were prepared as follows: juice $(5 \mathrm{~mL})$ was poured in a SPME vial containing $\mathrm{NaCl}(3 \mathrm{~g})$ and deuterated standards $\left(50 \mu \mathrm{L}\right.$ of a mixture of ethyl acetate- $\mathrm{d}_{8}$, ethyl butanoate-4,4,4- $\mathrm{d}_{3}$, benzyl-2,3,4,5,6- $\mathrm{d}_{5}$ alcohol, 2-phenyl- $\mathrm{d}_{5}-$ ethanol, hexanoic- $\mathrm{d}_{11}$ acid, ethyl octanoate- $\mathrm{d}_{15}$ and hexanol- $\mathrm{d}_{13}$ ) were added. Wine samples were prepared as follows: wine (3 $\mathrm{mL}$ ) was added to a SPME vial containing $\mathrm{NaCl}(3 \mathrm{~g})$, distilled water $(3 \mathrm{~mL})$ and a mixture of deuterated standards $(50 \mu \mathrm{L}$; same composition as above) was added. Volatile compounds were extracted by SPME (25 $\left.\mathrm{min} ; 60{ }^{\circ} \mathrm{C}\right)$ using a polydime thylsiloxane:divinylbenzene:carboxen fiber assembly $(2 \mathrm{~cm}$ grey fiber, Sigma-Aldrich, St. Louis, MO), and desorbed for $5 \mathrm{~min}$, in splitless mode, in the inlet of a GC (Agilent 6890 Series, Santa Clara, CA) attached to a time-of-flight mass spectrometer (Pegasus HT TOF-MS; Leco, St. Joseph, MI) and a computer with the Leco ChromaTOF software (Leco, St. Joseph, MI). Volatile compounds were separated on a DB wax GC column (polyethylene glycol, $60 \mathrm{~m} \times 0.25 \mathrm{~mm}$ i.d. $\times 0.25 \mu \mathrm{m}$ film thickness; SGE, Austin, TX), and quantified using deuterated standards and 11-point calibration curves (one for juice and one for wine) based on authentic standards, as detailed by Slegers et al [3].

\section{Statistical Analyses}

Analyses of variance (ANOVA) based on a mixed model were performed on all data using the Mixed procedure of the SAS software (Statistical Analysis System Institute, Cary, NC). Means were compared using a Tukey's test $(\mathrm{P} \leq 0.05)$. A principal component analysis (PCA) comparing juice and wine respective volatile compound profiles was performed using the Princomp procedure of SAS software. Variables were selected based both on their frequency among samples and on the results of the ANOVA (significant variables). The analysis of frequency was performed using the Freq procedure of the SAS software. Variables showing a frequency lower than $50 \%$ were not included in the PCA, unless they provided significant information on a group of data.

\section{Results and Discussion}

\section{Technological parameters of musts and wines}

The chemical balance of musts has a significant impact on the style and quality of wines. The most common markers for grape juice quality are TSS and TA. Their values largely depend of the grape variety, the growing conditions and berry ripening [17]. In general, the TSS concentration of grape varieties used for the production of dry white wines ranges from 15 to $25^{\circ}$ Brix [17]. In this study, Frontenac gris showed the highest TSS concentration at $23.2^{\circ}$ Brix (Table 1 ) whereas Seyval blanc had the lowest concentration, at $19.7^{\circ}$ Brix. These differences partly relate to the intrinsic characteristics of each grape variety, but low TSS concentration is also frequent in northern climate, sometimes because berry ripening cannot be much extended in Fall due to the risk of frost and/or the risk of Botrytis infections on berries, which typically occurs when berries tear following heavy rains [18].

Musts from Frontenac gris and Frontenac blanc showed the highest TA with respective values of 15.0 and $16.0 \mathrm{~g}$ tartaric ac. eq./L, whereas the lowest TA value was found in Seyval blanc, at $9.5 \mathrm{~g}$ tartaric ac. eq./L. The TA of white grapes varieties generally ranges between 4 and $9 \mathrm{~g}$ tartaric ac. eq./L [17]. The $\mathrm{pH}$ values of musts from all varieties ranged from 2.96 in Seyval blanc juice to 3.18 in Frontenac blanc, which are typical values for white grape varieties. IHV varieties are known to have higher TA and lower $\mathrm{pH}$ when compared to $V$. vinifera, especially when they are grown in cold climates $[2,18]$. In addition, $V$. riparia-based hybrid varieties such as Frontenac

Table 1: Composition of juice (total soluble solids (TSS, ${ }^{\circ}$ Brix), titratable acidity (TA, g tartaric ac. eq./L), pH, yeast assimilable nitrogen concentration (YAN, $\mathrm{mg} / \mathrm{L}$ ), and wine (alcohol concentration (\% v/v), titratable acidity (TA, g tartaric ac. eq./L), pH, glycerol concentration ( $\mathrm{g} / \mathrm{L})$, volatile acidity (VA, $\mathrm{mg}$ acetic ac. eq./L) in the white interspecific hybrid Vitis varieties Frontenac white, Frontenac gris, Seyval, Vidal St. Pepin harvested in Quebec during the 2012 season.

\begin{tabular}{|c|c|c|c|c|c|c|c|c|c|}
\hline \multirow[t]{2}{*}{ Variety } & \multicolumn{4}{|l|}{ Juice } & \multicolumn{5}{|l|}{ Wine } \\
\hline & $\begin{array}{l}\text { TSS } \\
\left({ }^{\circ} \text { Brix }\right)\end{array}$ & $\begin{array}{l}\text { TA } \\
\text { (g tartaric acid } \\
\text { eq. } / \mathrm{L} \text { ) }\end{array}$ & $\mathrm{pH}$ & $\begin{array}{l}\text { YAN } \\
(\mathrm{mg} / \mathrm{L})\end{array}$ & $\begin{array}{l}\text { Alcohol } \\
(\% \mathrm{v} / \mathrm{v})\end{array}$ & $\begin{array}{l}\mathrm{TA} \\
\text { (g tartaric } \\
\operatorname{acid} \text { eq./L) }\end{array}$ & $\mathrm{pH}$ & $\begin{array}{l}\text { Glycerol } \\
(\mathrm{g} / \mathrm{L})\end{array}$ & $\begin{array}{l}\text { VA } \\
\text { (mg acetic ac. } \\
\text { eq./L) }\end{array}$ \\
\hline Frontenac blanc & $22.3 \mathrm{ab}^{\mathrm{a}}$ & $16.0 \mathrm{~b}$ & $3.18 \mathrm{~b}$ & $304 \mathrm{ab}$ & $12.9 \mathrm{~b}$ & $10.5 \mathrm{a}$ & $3.08 \mathrm{a}$ & $1.36 \mathrm{a}$ & $0.23 \mathrm{ab}$ \\
\hline Frontenac gris & $23.2 \mathrm{~b}$ & $15.0 \mathrm{~b}$ & $3.14 \mathrm{~b}$ & $376 \mathrm{~b}$ & $12.6 \mathrm{ab}$ & $12.4 \mathrm{a}$ & $3.05 \mathrm{a}$ & $1.38 \mathrm{a}$ & $0.16 \mathrm{a}$ \\
\hline Seyval blanc & $19.7 \mathrm{a}$ & $9.50 \mathrm{a}$ & $2.96 \mathrm{a}$ & $183 \mathrm{ab}$ & $10.4 \mathrm{a}$ & $9.05 \mathrm{a}$ & $2.98 \mathrm{a}$ & $1.29 \mathrm{a}$ & $0.26 \mathrm{ab}$ \\
\hline St. Pepin & $20.3 \mathrm{ab}$ & $11.3 \mathrm{a}$ & $3.05 \mathrm{ab}$ & $275 \mathrm{ab}$ & $11.8 \mathrm{ab}$ & $9.15 \mathrm{a}$ & $3.03 \mathrm{a}$ & $1.45 \mathrm{a}$ & $0.21 \mathrm{ab}$ \\
\hline Vidal & $20.1 \mathrm{ab}$ & $10.6 \mathrm{a}$ & $3.15 \mathrm{~b}$ & $112 \mathrm{a}$ & $11.6 \mathrm{ab}$ & $8.53 \mathrm{a}$ & $3.12 \mathrm{a}$ & $1.30 \mathrm{a}$ & $0.47 \mathrm{~b}$ \\
\hline
\end{tabular}

a Values represent the mean of 3 to 5 biological replicates per variety. Values on the same column followed by different letters are significantly different according to Tukey's test $(\mathrm{P} \leq 0.05)$. 
Table 2: Concentration of free volatiles $(\mu \mathrm{g} / \mathrm{L})$ in juice of the white interspecific hybrid Vitis varieties Frontenac white, Frontenac gris, Seyval, Vidal St. Pepin harvested in Quebec during the 2012 season.

\begin{tabular}{|c|c|c|c|c|c|}
\hline \multirow[t]{2}{*}{ Volatile Compound } & \multicolumn{5}{|c|}{ Concentration $(\mu \mathrm{g} / \mathrm{L})^{\mathrm{a}}$} \\
\hline & Frontenac blanc & Frontenac gris & Seyval & St. Pepin & Vidal \\
\hline \multicolumn{6}{|c|}{ Fatty acids degradation products } \\
\hline hexanal & $220 \pm 26.1$ & $47.2 \pm 44.3$ & $33.5 \pm 21.6$ & $22.6 \pm 22$ & $59.1 \pm 63.2$ \\
\hline hexanol & $196 \pm 57 \mathrm{a}$ & $153 \pm 46 a$ & $309 \pm 94 \mathrm{a}$ & $653 \pm 240 \mathrm{~b}$ & $383 \pm 130 \mathrm{a}$ \\
\hline cis-3-hexenal & $16.5 \pm 12.2 \mathrm{a}$ & $53.8 \pm 25.1 \mathrm{~b}$ & $20.3 \pm 15.5 \mathrm{a}$ & $13.7 \pm 7.1 \mathrm{a}$ & $14.8 \pm 11 \mathrm{a}$ \\
\hline cis-3-hexenol & $41 \pm 13.1 \mathrm{a}$ & $37.9 \pm 16.3 \mathrm{a}$ & $50.3 \pm 35.8 \mathrm{a}$ & $48.1 \pm 23.7 \mathrm{a}$ & $170 \pm 113 \mathrm{~b}$ \\
\hline trans-2-hexenal & $690 \pm 456 \mathrm{ab}$ & $965 \pm 372 b$ & $450 \pm 232 \mathrm{ab}$ & $308 \pm 350 \mathrm{a}$ & $394 \pm 209 \mathrm{ab}$ \\
\hline trans-2-hexenol & $994 \pm 179 \mathrm{c}$ & $830 \pm 198 \mathrm{bc}$ & $504 \pm 131 \mathrm{ab}$ & $986 \pm 249 \mathrm{c}$ & $435 \pm 158 \mathrm{a}$ \\
\hline trans, cis-2,6-nonadienal & $0.29 \pm 0.11 \mathrm{ab}$ & $0.36 \pm 0.14 b$ & $0.13 \pm 0.1 \mathrm{ab}$ & $0.08 \pm 0.17 \mathrm{a}$ & $0.04 \pm 0.09 \mathrm{a}$ \\
\hline trans, trans-2,4-hexadienal & $32.5 \pm 26.1$ & $38 \pm 17.5$ & $11.1 \pm 11.2$ & $12.1 \pm 13.7$ & $19.3 \pm 10$ \\
\hline heptanol & $t r$ & $0.02 \pm 0.01$ & $0.42 \pm 0.9$ & $0.36 \pm 0.68$ & tr \\
\hline trans-2-heptenal & $1.58 \pm 1.32$ & $1.44 \pm 0.73$ & $1.91 \pm 1.32$ & $1.39 \pm 1.77$ & $1.04 \pm 1.11$ \\
\hline trans, cis-2,4-heptadienal & $0.48 \pm 0.13$ & $0.5 \pm 0.14$ & $0.37 \pm 0.21$ & $0.34 \pm 0.06$ & $0.34 \pm 0.09$ \\
\hline trans, trans-2,4-heptadienal & $2.43 \pm 0.61$ & $2.43 \pm 0.69$ & $1.9 \pm 1.08$ & $1.98 \pm 0.46$ & $1.74 \pm 0.23$ \\
\hline 1-octen-3-ol & $1.05 \pm 0.63$ & $0.83 \pm 0.35$ & $1.22 \pm 1.56$ & $3.38 \pm 5.74$ & $1.55 \pm 1.13$ \\
\hline 1-octen-3-one & $0.1 \pm 0.17$ & $0.01 \pm 0.01$ & $0.51 \pm 0.53$ & $0.32 \pm 0.62$ & $0 \pm 0.01$ \\
\hline 2-octanone & $0.1 \pm 0.1$ & $0.06 \pm 0.05$ & $0.27 \pm 0.41$ & $0.6 \pm 0.84$ & $0.07 \pm 0.01$ \\
\hline decanal & $0.47 \pm 0.55$ & $0.94 \pm 0.01$ & $0.75 \pm 0.42$ & $0.72 \pm 0.48$ & $0.55 \pm 0.5$ \\
\hline 2-undecanone & $0.4 \pm 0.34$ & $0.28 \pm 0.19$ & $0.76 \pm 0.8$ & $0.89 \pm 0.56$ & tr \\
\hline Sum & $2000 \pm 634 a$ & $2131 \pm 475 a$ & $1498 \pm 216 a$ & $2052 \pm 83 a$ & $1480 \pm 466 a$ \\
\hline \multicolumn{6}{|l|}{$C_{13}$-norisoprenoids } \\
\hline$\beta$-damascenone & $5.45 \pm 2.62 \mathrm{a}$ & $2.94 \pm 1.07 \mathrm{a}$ & $3.24 \pm 1.78 \mathrm{a}$ & $17.6 \pm 7.1 \mathrm{~b}$ & $6.86 \pm 4.43 \mathrm{~b}$ \\
\hline$\alpha$-ionone & $0.47 \pm 0.18 \mathrm{a}$ & $0.32 \pm 0.08 \mathrm{a}$ & $0.7 \pm 0.63 \mathrm{ab}$ & $1.39 \pm 0.5 \mathrm{ab}$ & $2.19 \pm 1.44 \mathrm{~b}$ \\
\hline$\alpha$-ionol & $1.73 \pm 0.7 \mathrm{a}$ & $1.39 \pm 0.28 \mathrm{a}$ & $2.72 \pm 1.86 \mathrm{ab}$ & $4.85 \pm 1.61 \mathrm{~b}$ & $1.33 \pm 0.79 \mathrm{a}$ \\
\hline$\beta$-ionone & $0.11 \pm 0.01$ & $0.1 \pm 0.01$ & $0.08 \pm 0.01$ & $0.1 \pm 0.03$ & $0.1 \pm 0.02$ \\
\hline \multicolumn{6}{|l|}{ Terpenes $^{b}$} \\
\hline$\beta$-myrcene & $1.4 \pm 0.93$ & $1.39 \pm 0.93$ & $1.87 \pm 0.09$ & $1.46 \pm 0.97$ & $2.57 \pm 4.31$ \\
\hline (R)-(+)-limonene & $0.12 \pm 0.03 \mathrm{a}$ & $0.13 \pm 0.01 \mathrm{a}$ & $0.14 \pm 0.07 \mathrm{a}$ & $0.28 \pm 0.18 \mathrm{a}$ & $2.29 \pm 1.22 \mathrm{~b}$ \\
\hline linalool & $0.99 \pm 0.06 \mathrm{a}$ & $1.02 \pm 0.14 \mathrm{a}$ & $2.37 \pm 1.27 \mathrm{a}$ & $3.11 \pm 0.68 \mathrm{ab}$ & $10.3 \pm 8.1 \mathrm{~b}$ \\
\hline$\alpha$-terpineol & $0.02 \pm 0.01 \mathrm{a}$ & $0.02 \pm 0.01 \mathrm{a}$ & $4.93 \pm 5.51 \mathrm{a}$ & $22.3 \pm 17.6 \mathrm{ab}$ & $38.2 \pm 14.5 \mathrm{~b}$ \\
\hline nerol & $n d$ & $0.01 \pm 0.02$ & $0.07 \pm 0.13$ & $0.04 \pm 0.04$ & $0.26 \pm 0.38$ \\
\hline Sum & $2.53 \pm 0.99 a$ & $2.57 \pm 1 a$ & $9.38 \pm 6.54 a$ & $27.2 \pm 17.1 a b$ & $53.6 \pm 27.4 \mathrm{~b}$ \\
\hline \multicolumn{6}{|l|}{ Fatty acid ethyl esters $^{b}$} \\
\hline ethyl propanoate & $\operatorname{tr}$ & $0.06 \pm 0.11$ & $n d$ & $1.01 \pm 0.44$ & $0.89 \pm 1.99$ \\
\hline ethyl 2-methylpropanoate & $n d$ & $0.21 \pm 0.42$ & $n d$ & $n d$ & nd \\
\hline ethyl butanoate & $t r$ & nd & $n d$ & $20.3 \pm 11.7$ & $n d$ \\
\hline ethyl 3-methylbutanoate & $n d$ & $n d$ & nd & $0.17 \pm 0.2$ & $n d$ \\
\hline ethyl 2-butenoate & $t r$ & $t r$ & $0.1 \pm 0.11 \mathrm{a}$ & $11.5 \pm 10 \mathrm{~b}$ & $n d$ \\
\hline ethyl hexanoate & $t r$ & $t r$ & $0.05 \pm 0.08$ & $0.42 \pm 0.82$ & $t r$ \\
\hline Sum & $t r$ & $0.27 \pm 0.39 a$ & $0.15 \pm 0.14 a$ & $33.5 \pm 22 b$ & $0.89 \pm 1.99 \mathrm{a}$ \\
\hline \multicolumn{6}{|c|}{ Volatile phenols and benzene derivatives } \\
\hline 2-phenylacetaldehyde & $0.89 \pm 1.62 \mathrm{a}$ & $0.8 \pm 1.3 \mathrm{a}$ & $2.55 \pm 4.58 \mathrm{a}$ & $2.88 \pm 3.97 \mathrm{a}$ & $54.4 \pm 46.1 \mathrm{~b}$ \\
\hline phenethyl acetate & tr & $\operatorname{tr}$ & $0.06 \pm 0.09$ & $0.07 \pm 0.02$ & $0.14 \pm 0.29$ \\
\hline 2-phenylethanol & $3.88 \pm 7.52$ & $2.79 \pm 5.39$ & $3.79 \pm 8.25$ & $0.8 \pm 0.78$ & $39 \pm 60.1$ \\
\hline eugenol & $0.52 \pm 0.36$ & $0.43 \pm 0.29$ & nd & $0.12 \pm 0.25$ & nd \\
\hline$p$-vinylguaiacol & $3.98 \pm 1.71 \mathrm{c}$ & $1.45 \pm 0.56 \mathrm{ab}$ & $0.74 \pm 0.48 \mathrm{ab}$ & $0.41 \pm 0.29 \mathrm{a}$ & $3.96 \pm 3.64 \mathrm{bc}$ \\
\hline Sum & $9.28 \pm 10.2 a$ & $5.48 \pm 5.56 a$ & $7.13 \pm 12.9 a$ & $4.28 \pm 4.7 a$ & $97.5 \pm 51.6 b$ \\
\hline
\end{tabular}




\begin{tabular}{|l|l|l|l|l|l|}
\hline Others \\
\hline isoamyl acetate & $0.03 \pm 0.06$ & $0.03 \pm 0.07$ & $0.19 \pm 0.24$ & $0.36 \pm 0.54$ \\
\hline isoamyl alcohol & $t r$ & $n d$ & $t r$ & $0.04 \pm 0.06$ & $0.04 \pm 0.08$ \\
\hline
\end{tabular}

${ }^{a}$ Values represent the mean \pm standard deviation of 3 to 5 biological replicates per variety. Values on the same line followed by different letters are significantly different according to Tukey's test $(\mathrm{P} \leq 0.05)$. Values on the same line without letters are not significantly different according to Tukey's test $(\mathrm{P} \leq 0.05)$.

${ }^{b}$ Ethyl 2 -methybutanoate and $\beta$-citronellol were not detected in any sample; ethyl octanoate was found in trace in all varieties but not all samples. nd: not detected

tr: compound found below the limit of quantification in most samples.

gris and Frontenac blanc typically show high TA due to their high concentration of tartaric acid $[3,18]$. High TA may be adjusted by deacidification, which is common practice in the production of cold-climate wine $[2,3]$. Indeed, $\mathrm{pH}$ and TA corrections are typical of wine production, regardless of the climate $[6,17]$.

A sufficient concentration of YAN $(\geq 150 \mathrm{mg} / \mathrm{L})$ in musts is essential to prevent stuck fermentations in winemaking. The YAN concentration of IHV varieties can be highly variable (between $8 \mathrm{mg} / \mathrm{L}$ and $928 \mathrm{mg} / \mathrm{L}$ ) and higher cold tolerance in grape varieties generally results in higher nitrogen concentration [19]. The must from Frontenac gris, one of the most cold-hardy varieties among those analyzed, contained the highest YAN concentration $(376 \mathrm{mg} / \mathrm{L}$, Table 1) whereas Vidal, which typically needs winter protection in Quebec, had the lowest YAN concentration $(112 \mathrm{mg} / \mathrm{L})$. Such low YAN values in Vidal musts resulted in slower fermentations and may explain the slightly higher VA concentration found in Vidal wine samples $(0.47 \mathrm{mg}$ acetic acid eq./L). The production of VA by yeast is inversely proportional to the amount of YAN present in the must [20].

\section{Volatile compounds from musts and wine}

Volatile compounds (40) from five chemical and metabolic classes were quantified in musts: 1) $\mathrm{C}_{6}$ compounds and other fatty acid degradation products (FADP); 2) $\mathrm{C}_{13}{ }^{-}$ norisoprenoids; 3 ) terpenes; 4) fatty acid ethyl esters (FAEE); 5) Volatile phenols and other phenyl derivatives (Table 2). Analysis of the volatile compounds of wine resulted in identification and quantification of 36 volatile compounds including berry-related $\left(\mathrm{C}_{6}\right.$ compounds, terpenes, $\mathrm{C}_{13}$ norisoprenoids, volatile phenols) and fermentation-related (higher alcohols, aromatic and aliphatic esters and acetates, free fatty acids) compounds (Table 3 ).

\section{$\mathrm{C}_{6}$ compounds and other FADP}

Fatty acid degradation products (FADP) are the main volatile compounds found in the must of many grape varieties and mostly include six-carbons saturated and unsaturated alcohols and aldehydes such as hexanal and cis-3-hexenol [3]. FADP accounted for $99 \%$ of the volatile fraction of Frontenac gris, Frontenac blanc and Seyval blanc, whereas the proportion of FADP accounted for $96 \%$ and $90 \%$ of total quantified volatile compounds in the musts of St. Pepin and Vidal, respectively. In $V$. vinifera, $\mathrm{C}_{6}$ compounds constitute the largest proportion of free volatile compounds in the socalled "neutral" varieties and are significant contributors to the herbaceous character found in grape musts [21]. In agreement with the present work, previous studies have shown that $\mathrm{C}_{6}$ compounds are the main constituents of the flavor profile in several IHV varieties representing, in most cases, over 94\% of total volatile compounds $[3,22]$. The $\mathrm{C}_{6}$ aldehyde trans2-hexenal and the $\mathrm{C}_{6}$ alcohols trans-2-hexenol and hexanol constituted the major share of the total volatile composition in the musts of the studied IHV varieties, which is typical in grape juice $[3,18]$.

Hexanol was the major $\mathrm{C}_{6}$ compound found in the wines, and its concentration ranged from $391 \mu \mathrm{g} / \mathrm{L}$ in Frontenac blanc to $558 \mu \mathrm{g} / \mathrm{L}$ in Seyval blanc (Table 3), suggesting that its sensory impact is very limited since the odor perception threshold of hexanol is estimated at $8000 \mu \mathrm{g} / \mathrm{L}$ in wine [23] (Table 4). These values compare to those found in wines from $V$. vinifera varieties for which reported values ranged from 117 to $1101 \mu \mathrm{g} / \mathrm{L}$ in six grape varieties grown in Portugal, including Riesling (195 $\mu \mathrm{g} / \mathrm{L})$, and Sauvignon Blanc (390 $\mu \mathrm{g} / \mathrm{L})[24,25]$. Both the concentration and the sensory impact of $\mathrm{C}_{6}$ compounds are known to decrease in wine when compared to grape juice. Indeed, the highly odor-active $\mathrm{C}_{6}$ aldehydes found in grape juice are reduced to alcohol during winemaking, whereas specific $\mathrm{C}_{6}$ compounds are transformed into desirable esters and acetates [26]. $\mathrm{C}_{6}$ compounds from grapes may therefore contribute to fruity notes in wine [26].

However, $\mathrm{C}_{6}$ compounds and other FADP may also negatively impact wine aroma when their concentrations exceed their olfactory detection threshold [27]. In this study, $\mathrm{C}_{9}$ aldehydes nonanal and trans,cis-2,6-nonadienal showed odor activity values much higher than 1 ( 27 and 73 , in average, respectively), suggesting that they may have significantly impacted wine aroma (Table 4). Nonanal aroma is described as waxy with citrus and cucumber hints, whereas trans, cis-2,6nonadienal has a herbaceous scent with notes of cucumber and melon. The concentrations of nonanal determined in the wines ranged from $22.4 \mu \mathrm{g} / \mathrm{L}$ in Seyval blanc to $34.1 \mu \mathrm{g} / \mathrm{L}$ in St. Pepin (Table 3). In contrast, nonanal concentration has been reported to range from $1 \mu \mathrm{g} / \mathrm{L}$ [28] to $19 \mu \mathrm{g} / \mathrm{L}$ [29] in $V$. vinifera Chardonnay. Similarly, trans,cis-2,6-nonadienal is rarely reported in the volatile compound profile of wines from $V$. vinifera varieties; a recent study indicated that trans-2,cis-6nonadienal was not detected in any of 18 Spanish wines [28].

\section{Terpenes}

$\beta$-myrcene, linalool and $\alpha$-terpineol were the main terpenes quantified in the musts of the IHV varieties. Musts from Vidal grapes showed a significantly higher concentration in terpenes $(57.6 \mu \mathrm{g} / \mathrm{L})$, mainly linalool and $\alpha$-terpineol, compared to the other varieties (Table 2). The concentration of 
Table 3: Concentration of free volatiles ( $\mu \mathrm{g} / \mathrm{L}$, except where otherwise indicated) of wines from the white interspecific hybrid $V i t i s$ varieties Frontenac white, Frontenac gris, Seyval, Vidal St. Pepin harvested in Quebec during the 2012 season.

\begin{tabular}{|c|c|c|c|c|c|}
\hline \multirow[t]{2}{*}{ Volatile compound } & \multicolumn{5}{|l|}{ Concentration $^{\mathrm{a}, \mathrm{b}}$} \\
\hline & Frontenac blanc & Frontenac gris & Seyval & St. Pepin & Vidal \\
\hline \multicolumn{6}{|c|}{ Fatty acids degradation products } \\
\hline hexanal & $2.98 \pm 0.93$ & $2.36 \pm 0.59$ & $2.62 \pm 2.4$ & $3.95 \pm 2.51$ & $1.21 \pm 1.06$ \\
\hline hexanol & $391 \pm 201$ & $491 \pm 52$ & $558 \pm 136$ & $476 \pm 59$ & $394 \pm 51$ \\
\hline trans-3-hexenol & $18.2 \pm 3.7 \mathrm{ab}$ & $13.7 \pm 2.9 \mathrm{ab}$ & $11.1 \pm 5 \mathrm{ab}$ & $22.3 \pm 8.4 \mathrm{~b}$ & $7.5 \pm 2.23 \mathrm{a}$ \\
\hline cis-3-hexenol & $27 \pm 8.6 \mathrm{a}$ & $35 \pm 8.7 \mathrm{a}$ & $57.7 \pm 25 \mathrm{a}$ & $31 \pm 12 \mathrm{a}$ & $144.9 \pm 26.3 b$ \\
\hline nonanal & $27.4 \pm 10.1$ & $29.4 \pm 5.1$ & $22.4 \pm 5.6$ & $34.1 \pm 10.5$ & $25.5 \pm 3.5$ \\
\hline trans,cis-2,6-nonadienal & $0.26 \pm 0.45$ & $1.53 \pm 2.66$ & $0.55 \pm 0.84$ & $0.71 \pm 0.91$ & $0.62 \pm 1.07$ \\
\hline Sum & $467 \pm 197$ & $573 \pm 49$ & $652 \pm 118$ & $567 \pm 51$ & $573 \pm 38$ \\
\hline \multicolumn{6}{|l|}{$C_{13}$-norisoprenoids } \\
\hline$\beta$-damascenone & $2.67 \pm 0.24$ & $2.99 \pm 0.82$ & $0.82 \pm 0.77$ & $2.66 \pm 1.68$ & $2.23 \pm 0.3$ \\
\hline \multicolumn{6}{|l|}{ Terpenes } \\
\hline$\beta$-myrcene & $t r$ & $t r$ & $t r$ & $t r$ & $t r$ \\
\hline$p$-cymenene & $0.57 \pm 0.61 \mathrm{ab}$ & $0.5 \pm 0.71 \mathrm{ab}$ & $0.51 \pm 0.72 \mathrm{a}$ & $0.44 \pm 0.29 \mathrm{a}$ & $2.05 \pm 0.68 b$ \\
\hline linalool & $7.28 \pm 3.65 \mathrm{a}$ & $6.99 \pm 0.69 \mathrm{a}$ & $8.36 \pm 7.08 \mathrm{a}$ & $10.2 \pm 9.3 \mathrm{ab}$ & $23.4 \pm 1.16 b$ \\
\hline$\alpha$-terpineol & $2.24 \pm 0.44$ & $3.95 \pm 0.92$ & $4.61 \pm 2.44$ & $10.6 \pm 11.01$ & $12.3 \pm 1.64$ \\
\hline$\beta$-citronellol & $6.67 \pm 2.94 \mathrm{a}$ & $6.99 \pm 0.34 \mathrm{a}$ & $4.26 \pm 2.46 \mathrm{a}$ & $5.91 \pm 2.2 \mathrm{a}$ & $14.4 \pm 4.7 \mathrm{~b}$ \\
\hline nerol & $n d$ & nd & nd & $n d$ & $1.09 \pm 0.05$ \\
\hline geraniol & $0.13 \pm 0.17$ & $n d$ & $t r$ & $1.88 \pm 3.76$ & $1.74 \pm 0.68$ \\
\hline Sum & $16.9 \pm 6.5 a$ & $18.4 \pm 1 a$ & $17.7 \pm 5.9 a$ & $29 \pm 25.4 a b$ & $55 \pm 4.5 b$ \\
\hline \multicolumn{6}{|l|}{ Fatty acid ethyl esters } \\
\hline ethyl 2-methylpropanoate & $213 \pm 21$ & $193 \pm 57$ & $223 \pm 41$ & $274 \pm 40$ & $188 \pm 9$ \\
\hline ethyl butanoate & $164 \pm 48$ & $157 \pm 55$ & $79 \pm 122$ & $115 \pm 95$ & $112 \pm 29$ \\
\hline ethyl 2-methylbutanoate & $15.2 \pm 2.6 \mathrm{ab}$ & $23.3 \pm 9 \mathrm{ab}$ & $27.4 \pm 4.3 b$ & $24.7 \pm 8.2 \mathrm{~b}$ & $8.7 \pm 3.4 \mathrm{a}$ \\
\hline ethyl 3-methylbutanoate & $20.1 \pm 2.8 \mathrm{ab}$ & $25.1 \pm 4 b$ & $21.2 \pm 3.2 \mathrm{~b}$ & $21.7 \pm 5.2 \mathrm{~b}$ & $10.9 \pm 2.3 \mathrm{a}$ \\
\hline ethyl hexanoate & $2274 \pm 1192$ & $1628 \pm 586$ & $1449 \pm 828$ & $1911 \pm 262$ & $2598 \pm 568$ \\
\hline ethyl octanoate & $6544 \pm 4243$ & $5170 \pm 3666$ & $5107 \pm 3526$ & $6030 \pm 1101$ & $12089 \pm 3553$ \\
\hline ethyl decanoate & $1752 \pm 1560 \mathrm{a}$ & $3991 \pm 2450 \mathrm{a}$ & $4833 \pm 3238 \mathrm{a}$ & $4215 \pm 2030 \mathrm{a}$ & $12264 \pm 3779 b$ \\
\hline ethyl 3-hydroxyhexanoate & $6.7 \pm 2.1$ & $9.6 \pm 4.6$ & $7 \pm 4.6$ & $11.3 \pm 3.4$ & $8.5 \pm 6.8$ \\
\hline $\operatorname{Sum}(m g / L)$ & $11.0 \pm 4.3 a$ & $11.2 \pm 6.6 a$ & $11.7 \pm 7.7 a$ & $12.6 \pm 2.3 a b$ & $27.3 \pm 7.8 b$ \\
\hline \multicolumn{6}{|l|}{ Aromatic esters } \\
\hline phenethyl acetate & $20.1 \pm 19.1 \mathrm{a}$ & $27.3 \pm 34.8 \mathrm{a}$ & $62.7 \pm 45.3 \mathrm{ab}$ & $105 \pm 17 \mathrm{bc}$ & $150 \pm 13.8 \mathrm{c}$ \\
\hline ethyl dihydrocinnamate & $t r$ & $t r$ & $t r$ & $0.06 \pm 0.1$ & $t r$ \\
\hline ethyl cinnamate & nd & nd & nd & $0.05 \pm 0.09$ & nd \\
\hline ethyl vanillate & $4.95 \pm 1.47 \mathrm{~b}$ & $5.21 \pm 2.58 \mathrm{~b}$ & $2.7 \pm 2.07 \mathrm{ab}$ & $1.29 \pm 1.37 \mathrm{a}$ & $1.07 \pm 0.85 \mathrm{a}$ \\
\hline Sum & $25.1 \pm 19.4 a$ & $32.6 \pm 33.9 a$ & $65.5 \pm 45 a b$ & $107 \pm 18 b c$ & $151 \pm 13 c$ \\
\hline \multicolumn{6}{|l|}{ Fatty acids } \\
\hline hexanoic acid (mg/L) & $6.35 \pm 1.96 \mathrm{a}$ & $5.16 \pm 2.29 \mathrm{a}$ & $5.96 \pm 2.77 \mathrm{a}$ & $6.86 \pm 1.56 \mathrm{a}$ & $12.2 \pm 1.2 \mathrm{~b}$ \\
\hline octanoic acid (mg/L) & $14.4 \pm 2.35 \mathrm{a}$ & $15.5 \pm 4.9 \mathrm{a}$ & $20 \pm 9.9 \mathrm{a}$ & $20.5 \pm 2.5 \mathrm{a}$ & $38.1 \pm 1.7 \mathrm{~b}$ \\
\hline $\operatorname{Sum}(m g / L)$ & $20.8 \pm 0.5 a$ & $20.6 \pm 0.7 a$ & $26 \pm 1.2 a$ & $27.4 \pm 4 a$ & $50.3 \pm 1.7 b$ \\
\hline \multicolumn{6}{|l|}{ Volatile phenols } \\
\hline eugenol & $1.16 \pm 0.47 \mathrm{ab}$ & $2.17 \pm 0.74 \mathrm{~b}$ & $0.7 \pm 0.31 \mathrm{a}$ & $0.5 \pm 0.38 \mathrm{a}$ & $0.17 \pm 0.17 \mathrm{a}$ \\
\hline$p$-vinylguaiacol & $14.9 \pm 12.2 \mathrm{ab}$ & $25 \pm 11 \mathrm{ab}$ & $10.1 \pm 8.4 \mathrm{a}$ & $19.6 \pm 16.3 \mathrm{ab}$ & $42.8 \pm 6.2 \mathrm{~b}$ \\
\hline Sum & $16.1 \pm 12.3 a b$ & $27.1 \pm 11.7 a b$ & $10.8 \pm 8.4 a$ & $20.1 \pm 16.5 a b$ & $43 \pm 6.2 b$ \\
\hline \multicolumn{6}{|c|}{ Other fermentation compounds } \\
\hline isobutyl acetate & $0.47 \pm 0.41$ & nd & $0.35 \pm 0.44$ & nd & nd \\
\hline isobutanol & $162 \pm 26 \mathrm{ab}$ & $168 \pm 42 b$ & $125 \pm 18 \mathrm{ab}$ & $161 \pm 18 b$ & $95 \pm 29 \mathrm{a}$ \\
\hline
\end{tabular}




\begin{tabular}{|l|l|l|l|l|l|}
\hline isoamyl acetate & $598 \pm 452$ & $331 \pm 537$ & $335 \pm 300$ & $766 \pm 324$ \\
\hline hexyl acetate & $4.24 \pm 4.05 \mathrm{a}$ & $1.67 \pm 1.6 \mathrm{a}$ & $9.71 \pm 7.63 \mathrm{ab}$ & $13.6 \pm 3.7 \mathrm{ab}$ \\
\hline acetoin $(\mathrm{mg} / \mathrm{L})$ & $16.1 \pm 18.4$ & $12.2 \pm 5.4$ & $4.02 \pm 4.37$ & $22.3 \pm 6.2 \mathrm{~b}$ & $2.97 \pm 1.99$ \\
\hline ethyl lactate $(\mathrm{mg} / \mathrm{L})$ & $31.2 \pm 42.3$ & $27.2 \pm 25.2$ & $13.1 \pm 18.8$ & $5.79 \pm 1.45$ & $6.05 \pm 2.31$ \\
\hline butyrolactone & $263 \pm 12$ & $277 \pm 8$ & $287 \pm 44$ & $22.2 \pm 33.8$ & $257 \pm 12$ \\
\hline 2-phenylethanol $(\mathrm{mg} / \mathrm{L})$ & $34.8 \pm 7.3$ & $43.9 \pm 17.4$ & $54.9 \pm 11.7$ & $54.8 \pm 14.2$ & $50.7 \pm 11.6$ \\
\hline
\end{tabular}

${ }^{a}$ Concentrations are in $\mu \mathrm{g} / \mathrm{L}$, unless otherwise indicated.

${ }^{\mathrm{b}}$ Values represent the mean \pm standard deviation of 3 to 5 biological replicates per variety. Values on the same line followed by different letters are significantly different according to Tukey's test $(\mathrm{P} \leq 0.05)$. Values on the same line without letters are not significantly different according to Tukey's test $(\mathrm{P} \leq 0.05)$.

nd: not detected

tr: compound found below the limit of quantification in most samples.

linalool $(10.3 \mu \mathrm{g} / \mathrm{L})$ measured in Vidal must is comparable to those previously observed in neutral $V$. vinifera white varieties, whereas its $\alpha$-terpineol concentration $(38.2 \mu \mathrm{g} / \mathrm{L})$ better relates to the amounts reported in aromatic $V$. vinifera varieties. Indeed, previous studies showed linalool and $\alpha$-terpineol concentrations ranging from 64.2 to $198 \mu \mathrm{g} / \mathrm{L}$, and from 9.5 to $37 \mu \mathrm{g} / \mathrm{L}$, respectively, in grape varieties described as "aromatic" $[30,31]$. Similar to most grape varieties analyzed in the present studies, the neutral grape varieties Albillo [30], Bical [31], Fiano [32], and Palomino Fino [33] have been reported to contain low concentrations of linalool (0 to 6.7 $\mu \mathrm{g} / \mathrm{L})$ and $\alpha$-terpineol (0 to $3.2 \mu \mathrm{g} / \mathrm{L})$.

Consistent with the profile of must volatile compounds, Vidal wines showed the highest terpene concentration (55.0 $\mu \mathrm{g} / \mathrm{L}$, Table 3). Terpene concentration ranged from $17 \mu \mathrm{g} / \mathrm{L}$ in Frontenac blanc to $29 \mu \mathrm{g} / \mathrm{L}$ in St. Pepin. Linalool, $\alpha$-terpineol and $\beta$-citronellol were the main terpenes found in the wines. The concentration of linalool in Vidal wine $(23 \mu \mathrm{g} / \mathrm{L})$ compared with reported values in Riesling (16 $\mu \mathrm{g} / \mathrm{L})$ [24]. Terpenes are known to bring desirable floral and citrus aroma in wine, and linalool has been identified as an impact odorant in young Vidal wine [10]. In this study, the odor-activity value for linalool in Vidal wine was $>1$ (Table 4), suggesting that this compound contributes to Vidal wine aroma.

\section{$\mathrm{C}_{13}$-norisoprenoids}

$\mathrm{C}_{13}$-norisoprenoids such as $\beta$-damascenone and $\beta$-ionone, contribute to the varietal aroma of many grape varieties due to their extremely low olfactory perception thresholds $(0.05$ and $0.09 \mu \mathrm{g} / \mathrm{L}$, respectively, Table 4) [34]. In this study, the concentrations of $\mathrm{C}_{13}$-norisoprenoids in grape musts ranged from $4.7 \mu \mathrm{g} / \mathrm{L}$ in Frontenac gris to $24.0 \mu \mathrm{g} / \mathrm{L}$ in St. Pepin, with the main compounds being $\beta$-damascenone and $\alpha$-ionol (Table 2). The variety St. Pepin showed the highest concentration of $\beta$-damascenone at $17.6 \mu \mathrm{g} / \mathrm{L}$, yet this compound concentration reached beyond its odor threshold in all varieties, suggesting that it significantly impacts the sensory aroma of juice from IHV varieties, as observed in $V$. vinifera and $V$. labrusca. $[35,36]$.

$\beta$-Damascenone has a fruity, floral scent reminiscent of baked apple, and has been reported to significantly contribute to the aroma of Vidal wine $[9,10]$. It was the main $\mathrm{C}_{13}{ }^{-}$ norisoprenoid quantified in IHV wines where its concentration ranged from $0.8 \mu \mathrm{g} / \mathrm{L}$ in Seyval blanc to $3.0 \mu \mathrm{g} / \mathrm{L}$ in Frontenac gris (Table 3 ). In contrast with musts, no significant differences between the varieties were found for $\beta$-damascenone. $\beta$-damascenone concentration decreases during winemaking due to reactions with sulfites and molecular rearrangements, especially in acidic conditions [37].

\section{Volatile phenols and other phenyl derivatives}

Volatile phenols occurring in grapes mostly derive from the shikimate metabolic pathway [34]. In this study, 2-phenylacetaldehyde and 2-phenylethanol were the main volatile phenols identified in IHV varieties, and had the highest concentrations in Vidal musts with values of 54 and $39 \mu \mathrm{g} / \mathrm{L}$, respectively (Table 2). A high concentration of 2-phenylacetaldehyde seems unusual in grape must since it has either not been detected ( $V$. labrusca, $V$. amurensis) or recovered in amounts ranging from traces in Cabernet Gernisht to 14 $\mu \mathrm{g} / \mathrm{L}$ in Muscadine $[22,35,38]$. However, recent work from our group showed very high concentrations (298 to $413 \mu \mathrm{g} / \mathrm{L}$ ) of 2-phenyacetaldehyde in the IHV Sabrevois and St. Croix, suggesting that certain varieties carry this particularity [3].

The main volatile phenol identified in IHV wines was $p$-vinylguaiacol. Other aromatic derivatives such as phenethyl acetate were also present in significant amounts (Table 3). Vidal wines showed the highest concentration in both $p$-vinylguaiacol $(42.8 \mu \mathrm{g} / \mathrm{L})$ and phenethyl acetate $(150 \mu \mathrm{g} / \mathrm{L}) . p$-Vinylguaiacol, whose flavor is reminiscent of spices and roasted peanuts, has an odor threshold of $40 \mu \mathrm{g} / \mathrm{L}$ in wines [23] suggesting that it may impact Vidal wine aroma $(\mathrm{OAV}>1$; Table 4$)$. $p$-Vinylguaiacol had been identified as an impact odorant of Vidal wine, but only at a concentration of $111 \mu \mathrm{g} / \mathrm{L}$ [9].

\section{Fatty acid ethyl esters and free fatty acids}

The concentration in fatty acid ethyl ester derivatives in must was insignificant in most IHV varieties analyzed, except in St. Pepin musts, where a significant concentration $(34 \mu \mathrm{g} / \mathrm{L}$, Table 2), and a larger variety of fatty acid ethyl ester structures were found. The main fatty acid esters found in St. Pepin were ethyl butanoate and the unsaturated ethyl 2-butenoate, but their concentration varied among St. Pepin samples, hence explaining the large standard deviations. The presence of ethyl esters is unusual in most grape varieties used in wine production but has been reported in American Vitis spp. such 
Table 4: Odor perception threshold $(\mathrm{OPT} ; \mu \mathrm{g} / \mathrm{L})$ and odor activity value $(\mathrm{OAV})$ of wines from the white interspecific hybrid Vitis varieties Frontenac white, Frontenac gris, Seyval, Vidal St. Pepin harvested in Quebec during the 2012 season.

\begin{tabular}{|c|c|c|c|c|c|c|}
\hline \multirow[b]{2}{*}{ Volatile compound } & \multirow{2}{*}{$\begin{array}{l}\text { OPT } \\
\text { (ref.) }\end{array}$} & \multicolumn{5}{|c|}{ OAV } \\
\hline & & $\begin{array}{l}\text { Frontenac } \\
\text { blanc }\end{array}$ & $\begin{array}{l}\text { Frontenac } \\
\text { gris }\end{array}$ & Seyval & $\begin{array}{l}\text { St. } \\
\text { Pepin }\end{array}$ & Vidal \\
\hline \multicolumn{7}{|c|}{ Fatty acids degradation products } \\
\hline hexanal & $5^{\mathrm{e}}$ & 0,6 & $<0.5$ & 0.5 & 1 & $<0.5$ \\
\hline hexanol & $8000^{b}$ & $<0.5$ & $<0.5$ & $<0.5$ & $<0.5$ & $<0.5$ \\
\hline trans-3-hexenol & $1000^{f}$ & $<0.5$ & $<0.5$ & $<0.5$ & $<0.5$ & $<0.5$ \\
\hline cis-3-hexenol & $400^{b}$ & $<0.5$ & $<0.5$ & $<0.5$ & $<0.5$ & $<0.5$ \\
\hline nonanal & $1^{g}$ & 27 & 29 & 22 & 34 & 25 \\
\hline $\begin{array}{l}\text { trans,cis-2,6-nona- } \\
\text { dienal }\end{array}$ & $0.01^{\mathrm{j}}$ & 26 & 153 & 55 & 71 & 62 \\
\hline \multicolumn{7}{|l|}{$C_{13}$-norisoprenoids } \\
\hline$\beta$-damascenone & $0.05^{b}$ & 53 & 60 & 16 & 53 & 45 \\
\hline \multicolumn{7}{|l|}{ Terpenes } \\
\hline$\beta$-myrcene & $14^{\mathrm{c}}$ & - & - & - & - & - \\
\hline p-cymenene & ND & - & - & - & - & - \\
\hline linalool & $25.2^{\mathrm{a}}$ & $<0.5$ & $<0.5$ & $<0.5$ & $<0.5$ & 1 \\
\hline$\alpha$-terpineol & $250^{b}$ & $<0.5$ & $<0.5$ & $<0.5$ & $<0.5$ & $<0.5$ \\
\hline$\beta$-citronellol & $100^{\mathrm{b}}$ & $<0.5$ & $<0.5$ & $<0.5$ & $<0.5$ & $<0.5$ \\
\hline nerol & $400^{k}$ & - & - & - & & $<0.5$ \\
\hline geraniol & $30^{\mathrm{a}}$ & $<0.5$ & - & - & $<0.5$ & $<0.5$ \\
\hline
\end{tabular}

\section{Fatty acid ethyl esters}

\begin{tabular}{|l|l|l|l|l|l|l||}
\hline ethyl 2-methylpropanoate & $15^{\text {a }}$ & 14 & 13 & 15 & 18 & 13 \\
\hline ethyl butanoate & $20^{\text {b }}$ & 8 & 8 & 4 & 6 & 6 \\
\hline
\end{tabular}

\begin{tabular}{|l|l|l|l|l|l|l||}
\hline ethyl butanoate & $20^{\mathrm{b}}$ & 8 & 8 & 4 & 6 & 6 \\
\hline ethyl 2-methylbutanoate & $18^{\mathrm{a}}$ & 1 & 1 & 2 & 1 & 0 \\
\hline ethyl 3-methylbutanoate & $3^{\mathrm{a}}$ & 7 & 8 & 7 & 7 & 4 \\
\hline ethyl hexanoate & $14^{\mathrm{a}}$ & 162 & 116 & 103 & 137 & 186 \\
\hline ethyl octanoate & $5^{\mathrm{a}}$ & 1309 & 1034 & 1021 & 1206 & 2418 \\
\hline ethyl decanoate & $200^{\mathrm{a}}$ & 9 & 20 & 24 & 21 & 61 \\
\hline ethyl 3-hydroxyhexanoate & $45^{\mathrm{h}}$ & $<0.5$ & $<0.5$ & $<0.5$ & $<0.5$ & $<0.5$ \\
\hline Aromatic esters & & & & & & \\
\hline phenethyl acetate & $250^{\mathrm{a}}$ & $<0.5$ & $<0.5$ & $<0.5$ & $<0.5$ & 1 \\
\hline $\begin{array}{l}\text { ethyl dihydrocinna- } \\
\text { mate }\end{array}$ & $1.6^{\mathrm{a}}$ & - & - & - & $<0.5$ & - \\
\hline ethyl cinnamate & $1.1^{\mathrm{a}}$ & - & - & - & $<0.5$ & - \\
\hline ethyl vanillate & $990^{\mathrm{i}}$ & $<0.5$ & $<0.5$ & $<0.5$ & $<0.5$ & $<0.5$ \\
\hline Fatty acids & & & & & & \\
\hline hexanoic acid (mg/L) & $0.42^{\mathrm{a}}$ & 15 & 12 & 14 & 16 & 29 \\
\hline octanoic acid (mg/L) & $0.5^{\mathrm{a}}$ & 29 & 31 & 40 & 41 & 76 \\
\hline Volatil phenols & & & & & & \\
\hline eugenol & $6^{\mathrm{a}}$ & $<0.5$ & $<0.5$ & $<0.5$ & $<0.5$ & $<0.5$ \\
\hline p-vinylguaiacol & $40^{\mathrm{b}}$ & $<0.5$ & 0.6 & $<0.5$ & 0.5 & 1 \\
\hline Other fermentation compounds & & & & & \\
\hline isobutyl acetate & $6140^{\mathrm{h}}$ & $<0.5$ & - & $<0.5$ & - & - \\
\hline
\end{tabular}


(PC 1, 40.8\% of variance) and trans-3-hexenol (PC 2, 15.6\% of variance) in wine, respectively. The presence of trans, cis-2,6nonadienal in wine was slightly related to the presence of this compound in the must, and was negatively correlated with the presence of terpenes in both must and wine over PC 1 . High concentrations of fatty acid ethyl esters in the must (PC 2), as found in St. Pepin, corresponded to higher concentration in nonanal in the wine.

Higher linalool concentration in the musts generally correlated with higher terpene concentrations in wine. However, fermentation significantly impacted the profile of terpenes found in wine, when compared to must. Indeed, certain terpene ( $\beta$-myrcene, $\alpha$-terpineol and nerol) decreased in the wine compared to the amount initially found in the must. Similarly, certain terpenes such as (R)-(+)-limonene were detected in musts but not in wines, whereas others such as geraniol, $p$-cymenene and $\beta$-citronellol were detected in wines but not in musts. Such changes may result from chemical rearrangements occurring during the winemaking process or during maturation in the bottle [25, 44]. For example, geraniol may be the precursor of other monoterpenes such as neral, geranial, nerol and $\beta$-citronellol, and that process may be affected by yeast in a specific way [44]. These rearrangements significantly impact wine aroma by modifying the odor perception threshold and the sensory descriptors of terpenes. On the other hand, the occurrence of many terpenes in wine results from the enzymatic or chemical hydrolysis of glycosylated precursors present in the must during the winemaking process [6].

Finally, both eugenol and $p$-vinylguaiacol showed a strong correlation between must and wine. Both of them increased in wine when compared to must, suggesting that non-volatile precursors may be present in the musts of IHV varieties. A similar relationship has been found in red IHV varieties [3]. Although glycosylated precursors of volatile phenols are present in grapes, the presence of vinyl phenols in white wine may result from the breakdown of $p$-cinnamic acid and ferulic acid [6].

\section{Conclusion}

The volatile compounds of musts and wines from hybrid grapes Frontenac gris, Frontenac blanc, Seyval, St. Pepin and Vidal grown in Quebec for the production of white wines was analyzed by GC-MS-SPME. Vidal showed the highest concentration in volatile compounds, as well as the widest chemical diversity in both must and wine. Vidal showed higher concentration of terpenes in musts and in wines, and fatty acid ethyl esters in its wines. On the contrary, Frontenac gris, Frontenac blanc, and Seyval blanc exhibited more neutral flavor profiles. The musts from St. Pepin showed the highest concentration of $\beta$-damascenone. PCA showed many correlations between must and wine: for instance, both $\mathrm{C}_{6}$ compounds and terpenes from musts were highly correlated with those later found in wine, suggesting that certain of these compounds could be used as markers for grape quality in the future. Knowledge gained on the volatile composition of these interspecific hybrid Vitis varieties will contribute to develop viticulture and winemaking practices that will optimize the

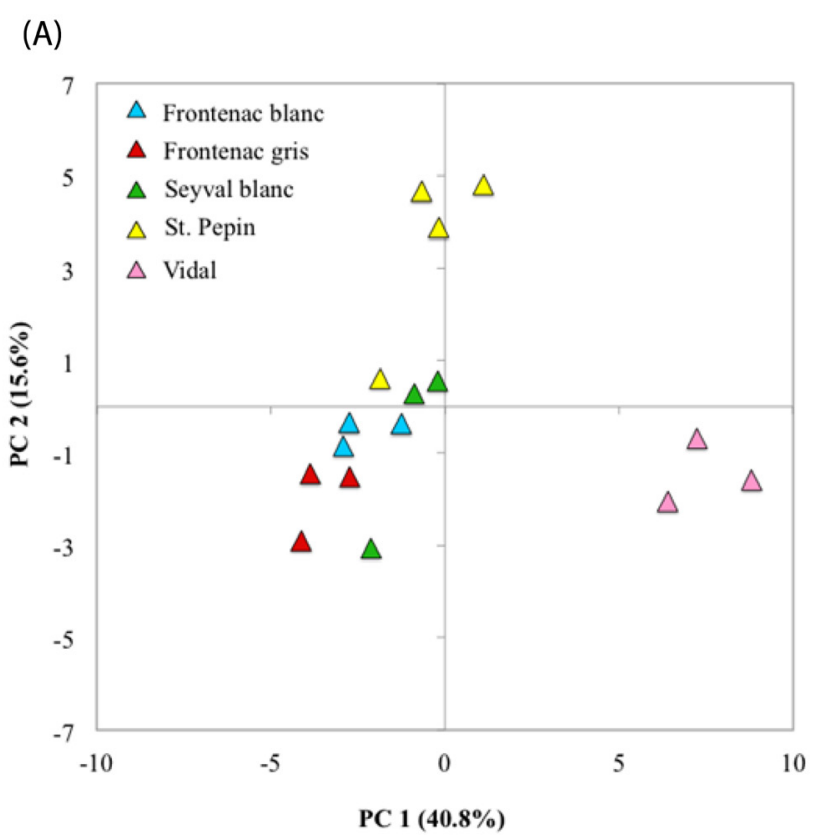

(B)

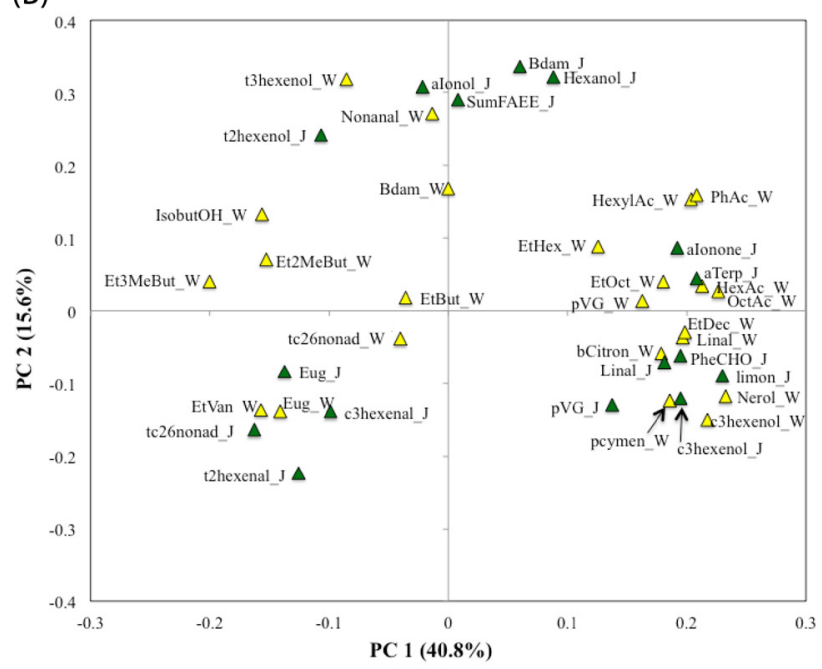

Figure 1: Principal Component Analysis (PCA) of the volatile composition of juice and wine from the interspecific hybrid Vitis varieties Frontenac blanc, Frontenac gris, Seyval blanc, St. Pepin and Vidal. (A) Biplot of varieties: Frontenac blanc (blue), Frontenac gris (red), Seyval blanc (green), St. Pepin (yellow) and Vidal (pink). (B) Biplot of variables, identified as follow: Juice volatile compounds (green triangles; labeled as _J): hexanol (Hexanol_J), cis3-hexenol (c3hexenol_J), trans-2-hexenol (t2hexenol_J), trans-2-hexenal (t2hexenal_J), trans,cis-2,6-nonadienal (tc26nonad_J), $\alpha$-ionol (aIonol_J), $\alpha$-ionone (aIonone $\mathrm{J}), \beta$-damascenone (Bdam $J \mathrm{~J}),(\mathrm{R})-(+)$-limonene (limon_J), $\alpha$-terpineol (aTerp_J), linalool (Linal_J), sum of fatty acid ethyl ester (FAEE_J), eugenol (Eug_J), $p$-vinylguaiacol ( $\left(\mathrm{VGG}_{-} \mathrm{J}\right), 2$-phenylacetaldehyde (PheCHO_J). Wine volatile compounds (yellow triangles; labeled as _W): trans-3-hexenol (t3hexenol_W), cis-3-hexenol (c3hexenol_W), nonanal (Nonanal_W), trans, cis-2,6-nonadienal (tc26nonad_W), $\beta$-damascenone (Bdam_W), $p$-cymenene (pcymen_W), linalool (Linal_W), $\beta$-citronellol (bCitron_W), nerol (Nerol_W), ethyl butanoate (EtBut_W), ethyl 2-methylbutanoate (Et2MeBut_W), ethyl 3-methylbutanoate (Et3MeBut_W), ethyl hexanoate (EtHex_W), ethyl octanoate (EtOct_W), ethyl decanoate (EtDec_W), phenylethyl acetate (PhAc_W), ethyl vanillate (EtVan_W), hexanoic acid (HexAc_W), octanoic acid (OctAc_W), eugenol (Eug_W), $p$-vinylguaiacol (pVG_W), isobutanol (isobutOH_W), hexyl acetate (HexylAc_W).

quality of their wines and maximize their potential on the global wine market. 


\section{Acknowledgments}

The authors thank the Ministère de l'Agriculture, des Pêcheries et de l'Alimentation (MAPAQ) from the Gouvernement du Québec, for financing this study. We also wish to thank the Association des vignerons du Québec for supporting the project, the wineries that participated in the study and the industry professionals (viticulturists, enologists, interns) who contributed to different extent to this research. In additions, we wish to acknowledge the contributions of interns/graduate students Catherine Barthe, Marie-Pascale Gagné, Louis Charron, Pauline Vautard, David Rochette, and Quentin Teulon, to grape sampling, winemaking and analysis.

\section{References}

1. MarketLine. 2012. Wine: Global Industry Almanac.

2. Pedneault K, Provost C. 2016. Fungus resistant grape varieties as a suitable alternative for organic wine production: benefits, limits, and challenges. Sci Hort 208: 57-77. doi: 10.1016/j.scienta.2016.03.016

3. Slegers A, Angers P, Ouellet É, Truchon T, Pedneault K. 2015. Volatile compounds from grape skin, juice and wine from five interspecific hybrid grapes cultivars grown in Québec (Canada) for wine production. Molecules 20(6): 10980-11016. doi: 10.3390/molecules200610980

4. Pollefeys P, Bousquet J. 2003. Molecular genetic diversity of the FrenchAmerican grapevine hybrids cultivated in North America. Genome 46(6): 1037-1048. doi: 10.1139/g03-076

5. Laurin F. 2015. État de la situation économique du secteur vitivinicole au Québec. Symposium Vigne et Vin.

6. Ribéreau-Gayon P, Glories Y, Maujean A, Dubourdieu, D. 2006. Handbook of enology, Volume 2: The chemistry of wine stabilization and treatments. John Wiley \& Sons, Ltd, Chichester, UK.

7. Jackson RS. 2008. Wine science: principles and applications. Academic Press, Boston, USA.

8. Moreno-Arribas MV, Polo MC. 2009. Wine chemistry and biochemistry. Springer, New York, USA.

9. Bowen AJ, Reynolds AG. 2012. Odor potency of aroma compounds in Riesling and Vidal blanc table wines and icewines by gas chromatography-olfactometry-mass spectrometry. J Agric Food Chem 60(11): 2874-2883. doi: 10.1021/jf203314j

10. Chisholm MG, Guiher LA, Zaczkiewlcz SM. 1995. Aroma characteristics of aged Vidal blanc wine. Am J Enol Vitic 46(1): 56-62.

11. Chisholm MG, Guiher LA, Vonah TM, Beaumont JL. 1994. Comparison of some French-American hybrid wines with white Riesling using gas chromatography-olfactometry. AmJ Enol Vitic 45(2): 201-212.

12. Skinkis PA, Bordelon BP, Wood KV.2008. Comparison of monoterpene constituents in Traminette, Gewürztraminer, and Riesling winegrapes. Am J Enol Vitic 59(4): 440-445.

13. Khanizadeh S, Rekika D, Levasseur A, Groleau Y, Richer C, et al. 2005. The effects of different cultural and environmental factors on grapevine growth, winter hardiness and performance in three locations in Canada. Small Fruits Review 4(3): 3-28. doi: 10.1300/J301v04n03_02

14. Khanizadeh S, Rekika D, Porgès L, Levasseur A, Groleau Y, et al. 2008. Soluble solids, acidity, canopy fruit distribution and disease susceptibility of selected grapes in Quebec. International Journal of Fruit Science 8(3): 200-215. doi: 10.1080/15538360802529781

15. Barthe C, Dorais M, Dubé G, Angers P, Pedneault K. 2013. Impact of cluster thinning on maturity and grape quality of Seyval blanc, an hybrid grape variety grown in Québec. Abstracts from the Presentations at the American Society for Enology and Viticulture-Eastern Section 38th Annual Conference, 15-18 July 2013, Winston-Salem, NC, USA. Am J
Enol Vitic 64: 416A-424A. doi: 10.5344/ajev.2013.ea

16. Amerine MA, Ough CS. 1980. Methods for analyses of musts and wines. John Wiley and Sons, New York, USA.

17. Jeffery D, Wilkinson K. 2014, Wine. In: Bamforth CW, Ward RE (eds) The Oxford handbook of food fermentations. Oxford University Press, New York, USA, pp 54-163.

18. Pedneault K, Dorais M, Angers P. 2013. Flavor of cold-hardy grapes: Impact of berry maturity and environmental conditions. J Agric Food Chem 61(44): 10418-10438. doi: 10.1021/jf402473u

19. Stewart ACH. 2013. Nitrogen composition of interspecific hybrid and Vitis vinifera wine grapes from the Eastern United States. Ph.D. Thesis, Purdue University, Indiana, USA.

20. Bely M, Rinaldi A, Dubourdieu D. 2003. Influence of assimilable nitrogen on volatile acidity production by Saccharomyces cerevisiae during high sugar fermentation. J Biosci Bioeng 96(6): 507-512. doi: 10.1016/S1389-1723(04)70141-3

21. Gómez E, Martínez A, Laencina J. 1995. Changes in volatile compounds during maturation of some grape varieties.J Sci Food Agric 67(2): 229-233. doi: 10.1002/jsfa.2740670213

22. Yang C, Wang Y, Liang Z, Fan P, Wu B, et al. 2009. Volatiles of grape berries evaluated at the germplasm level by headspaceSPME with GC-MS. Food Chem 114(3): 1106-1114. doi: 10.1016/j. foodchem.2008.10.061

23. Guth H. 1997. Quantification and sensory studies of character impact odorants of different white wine varieties. J Agric Food Chem 45(8): 3027-3032. doi: 10.1021/jf970280a

24. Vilanova M, Genisheva Z, Graña M, Oliveira JM. 2013. Determination of odorants in varietal wines from international grape cultivars (Vitis vinifera) grown in NW Spain. S Afr J Enol Vitic 34(2): 212-222.

25. Oliveira JM, Oliveira P, Baumes RL, Maia O. 2008. Changes in aromatic characteristics of Loureiro and Alvarinho wines during maturation. J Food Compos Anal 21(8): 695-707. doi: 10.1016/j. jfca.2008.08.002

26. Dennis EG, Keyzers RA, Kalua CM, Maffei SM, Nicholson EL, et al. 2012. Grape contribution to wine aroma: production of hexyl acetate, octyl acetate, and benzyl acetate during yeast fermentation is dependent upon precursors in the must. J Agric Food Chem 60(10): 2638-2646. doi: $10.1021 / \mathrm{jf} 2042517$

27. Joslin WS, Ough CS. 1978. Cause and fate of certain C6 compounds formed enzymatically in macerated grape leaves during harvest and wine fermentation. Am J Enol Vitic 29(1): 11-17.

28. Ferreira V, Culleré L, López R, Cacho J. 2004. Determination of important odor-active aldehydes of wine through gas chromatographymass spectrometry of their O-(2,3,4,5,6-pentafluorobenzyl)oximes formed directly in the solid phase extraction cartridge used for selective isolation. J Chromatogr A 1028(2): 339-345. doi: 10.1016/j. chroma.2003.11.104

29. Jiang B, Zhang Z. 2010. Volatile compounds of young wines from cabernet sauvignon, cabernet gernischet and chardonnay varieties grown in the loess plateau region of china. Molecules 15(12): 9184-9196. doi: $10.3390 /$ molecules15129184.

30. Sánchez Palomo E, Díaz-Maroto MC, González Viñas MA, SorianoPérez A, Pérez-Coello MS. 2007. Aroma profile of wines from Albillo and Muscat grape varieties at different stages of ripening. Food Control 18(5): 398-403. doi: 10.1016/j.foodcont.2005.11.006

31. Rocha S, Coutinho P, Barros A, Coimbra MA, Delgadillo I, et al. 2000. Aroma potential of two bairrada white grape varieties: Maria Gomes and Bical.J Agric Food Chem 48(10): 4802-4807. doi: 10.1021/ jf000175s

32. Ugliano M, Moio L. 2008. Free and hydrolytically released volatile compounds of Vitis vinifera L. cv. Fiano grapes as odour-active constituents of Fiano wine. Anal Cbim Acta 621(1): 79-85. doi: 10.1016/j.aca.2008.03.002 
33. Genovés S, Gil JV, Vallés S, Casas JA, Manzanares P. 2005. Assessment of the aromatic potential of Palomino Fino grape must using glycosidases. Am J Enol Vitic 56(2): 188-191.

34. Dunlevy JD, Kalua CM, Keyzers RA, Boss PK. 2009. The production of flavor and aroma compounds in grape berries. In: Roubelakis-Angelakis KA (ed) Grapevine molecular physiology \& biotechnology. Springer, Netherlands, pp 293-340.

35. Fan W, Xu Y, Jiang W, Li J. 2010. Identification and quantification of impact aroma compounds in 4 nonfloral Vitis vinifera varieties. J Food Sci 75(1): S81-S88. doi: 10.1111/j.1750-3841.2009.01436.x

36. Acree TE. 1981. The odor quality of Labrusca grapes. In: Teranishi R, Barerra-Benitez $\mathrm{H}$ (eds) Quality in selected fruits and vegetables of North America. American Chemical Society, Washington, DC, USA, pp 11-19. doi: 10.1021/bk-1981-0170.ch002

37. Sefton MA, Skouroumounis GK, Elsey GM, Taylor DK. 2011. Occurrence, sensory impact, formation and fate of damascenone in grapes, wines and other foods and beverages. J Agric Food Chem 59(18): 9717-9746. doi: 10.1021/jf201450q

38. Baek HH, Cadwallader KR, Marroquin E, Silva JL. 1997. Identification of predominant aroma compounds in Muscadine grape juice. J Food Sci 62(2): 249-252. doi: 10.1111/j.1365-2621.1997.tb03978.x

39. Schreier P, Paroschy JH. 1981. Volatile constituents from Concord, Niagara (Vitis labrusca, L.) and Elvira (V. labrusca, L. x V. riparia, M.) grapes. Canadian Institute of Food Science and Technology Journal 14(2): 112-118. doi: 10.1016/S0315-5463(81)72721-4

40. Ferreira V, Fernández P, Peña C, Escudero A, Cacho JF. 1995 Investigation on the role played by fermentation esters in the aroma of young Spanish wines by multivariate analysis. J Sci Food Agric 67(3): 381-392. doi: 10.1002/jsfa.2740670316

41. Culleré L, Escudero A, Cacho J, Ferreira V. 2004. Gas chromatographyolfactometry and chemical quantification study of the aroma of six premium quality Spanish aged red wines. J Agric Food Chem 52(6) 1653-1660. doi: 10.1021/jf0350820

42. Louw L, Tredoux AGJ, Van Rensburg P, Kidd M, Naes T, et al. 2010. Fermentation-derived aroma compounds in varietal young wines from South Africa. S Afr J Enol Vitic 31(2): 213-225.

43. Gallart M, Francioli S, Viu-Marco A, Lopez-Tamames E, Buxaderas S. 1997. Determination of free fatty acids and their ethyl esters in musts and wines. J Chromatogr A. 776(2): 283-291. doi: 10.1016/S00219673(97)00383-X

44. Gramatica P, Manitto B, Ranzi M, Delbianco A, Francavilla M. 1982. Stereospecific reduction of geraniol to (R)-(+)-citronellol by Saccharomyces cerevisiae. Experientia 38(7): 775-776. doi: 10.1007/ BF01972264

45. Ferreira V, López R, Cacho J. 2000. Quantitative determination of the odorants of young red wines from different grape varieties. J Sci Food Agric 80(11): 1659-1667. doi: 10.1002/1097-0010(20000901)80:11<1659::AID-JSFA693>3.0.CO;2

46. Buttery RG, Seifert BM, Guadagni DG, Black DR, Ling LC. 1968. Characterization of some volatile constituents of carrots. J Agric Food Chem 16(6): 1009-1015. doi: 10.1021/jf60160a012

47. Etievant PX. 1991. Wine. In: Maarse H (eds) Volatile compounds of food and beverages. Marcel Dekker, Inc., New York, USA, pp 483-533.

48. Buttery RG, Teranishi R, Flath RA, Ling LC. 1989. Fresh tomato volatiles: composition and sensory studies. In: Teranishi R, Buttery RG, Shahidi F (eds) Flavor chemistry: trends and developments. American Chemical Society, Washington, DC, USA, pp 211-222.

49. Hatanaka A, Kajiwara T, Horino H, Inokuchi K. 1992. Odor-structure relationships in n-hexenols and n-hexenals. Z Naturforsch C 47(3-4): 183-189.

50. Guadagni DG, Buttery RG, Okano S. 1963. Odour thresholds of some organic compounds associated with food flavours.J Sci Food Agric 14(10): 761-765. doi: 10.1002/jsfa.2740141014

51. Zea L, Moyano L, Moreno JA, Medina M. 2007. Aroma series as fingerprints for biological ageing in fino sherry-type wines. J Sci Food Agric 87(12): 2319-2326. doi: 10.1002/jsfa.2992

52. Campo E, Ferreira V, Escudero A, Marqués JC, Cacho J. 2006. Quantitative gas chromatography-olfactometry and chemical quantitative study of the aroma of four Madeira wines. Anal Chim Acta 563(1-2): 180-187. doi: 10.1016/j.aca.2005.10.035

53. Teranishi R, Buttery RG, Gaudagni DG. 1974. Odor quality and chemical structure in fruit and vegetable flavors. Annals N Y Acad Sci 237: 209-216. doi: 10.1111/j.1749-6632.1974.tb49855.x

54. Ribéreau-Gayon P, Boidron JN, Terrier A. 1975. Aroma of Muscat grape varieties. J Agric Food Chem 23(6): 1042-1047. doi: 10.1021/ jf60202a050 